\title{
A phase-based technique for localization of UHF-RFID tags moving on a conveyor belt: performance analysis and test-case measurements
}

\author{
A. Buffi, Member, IEEE, P. Nepa, Member, IEEE, F. Lombardini, Senior Member, IEEE
}

\begin{abstract}
A new phase-based technique for localization and tracking of items moving along a conveyor belt and equipped with UHF-RFID (Ultra High Frequency - Radio Frequency IDentification) tags is here described and validated. The technique is based on a synthetic-array approach that takes advantage of the fact that the tagged items move along a conveyor belt whose speed and path are known a priori. In this framework, a joint use is done of synthetic-array radar principles, knowledge-based processing, and efficient exploitation of the reader-tag communication signal. The technique can be easily implemented in any conventional reader based on an I$Q$ receiver and it does not require any modification of the reader antenna configurations usually adopted in UHF-RFID portals. Numerical results are used to investigate the performance analysis of such method, also furnishing system design guidelines. Finally, the localization capability is also demonstrated through a measurement campaign in a real conveyor belt scenario, showing that a centimeter order accuracy in the tag position estimation can be achieved even in a rich multipath environment.
\end{abstract}

Index Terms - UHF-RFID systems, RFID tag localization, phase-based localization techniques, synthetic array antenna.

\section{INTRODUCTION}

I $\mathrm{N}$ the last years, the development of more and more accurate localization radio technologies [1], [2] is attracting the academic and industrial research. Besides, Radio Frequency IDentification (RFID) technology [3] is gaining an increasing interest for authentication and management processes (e.g. logistics, anti-counterfeiting, access control, airport baggage management), as well as for localization and tracking of tagged items [4]. Therefore, a proliferation of localization and tracking solutions based on UHF-RFID systems is expected in the near future, to implement RFID-based Real Time Location Systems (RTLS) [5]. Localization techniques are required to exhibit a high robustness with respect to multipath and fixed/mobile clutter phenomena in typical crowded indoor scenarios. Also, one of the main objectives is to obtain a spatial resolution comparable to the size of the tagged items (from a few $\mathrm{cm}$ to tens of $\mathrm{cm}$ ), with minimal modifications of existing readers and antenna positioning/configuration (the latter aspect represents a must for limiting costs and reducing deployment time in scenarios where RFID technology is already implemented).

In this context, radiolocalization techniques can be of interest for a fully automatic management (i.e. transportation, routing, order checking and sorting) of UHF-RFID tagged items moving on a conveyor belt of airport handling systems, parcel distribution centers, and assembly lines with a high automation level. Depending on the specific application, a reliable spatial localization could be required, in addition to a simpler sorting operation. In some RFID portals mounted right on the conveyor belt, a possible localization approach consists in forcing the reader to read one tag at a time (without querying previous and next tags), by using reader antennas optimized to confine the electromagnetic field in a limited volume over the conveyor belt [6]-[11]. The detected tag will be just that one right under the RFID portal at the interrogation time and a correct sorting of all tagged items is made possible. However, the main advantage guaranteed from anti-collision algorithms implemented in UHF-RFID protocols, namely the simultaneous processing of all the tags that are inside the reader antenna beam, is lost. Indeed, each tag can be detected and localized only when it passes right under the reader portal (one-at-a-time technique). Radiolocalization algorithms

Manuscript received April 30, 2014; revised July 1, 2014; accepted July 23, 2014.

A. Buffi is with the Department of Information Engineering, University of Pisa, Pisa, Italy (corresponding author: phone: $+39-050-2217576$; fax: $+39-050-$ 2217522; e-mail: alice.buffi@iet.unipi.it).

P. Nepa and F. Lombardini are with the Department of Information Engineering, University of Pisa, Pisa, Italy (e-mail:, p.nepa@iet.unipi.it, f.lombardini@iet.unipi.it).

Copyright (c) 2014 IEEE. Personal use of this material is permitted. However, permission to use this material for any other purposes must be obtained from the IEEE by sending a request to pubs-permissions@ieee.org. 
exploiting detection information [12] or Received Signal Strength (RSS) information [13]-[15] can be also employed for tag localization on conveyor belts. In [15], the RSS data are employed together with the reading time stamps, the number of tag answers and the number of expected answers in a certain interval, for tag sorting in a conveyor belt scenario. Unfortunately, the performance of RSS-based localization techniques are quite limited in indoor scenarios, due to the difficulties in collecting reliable RSS values in rich multipath environments (multiple RSS peaks with comparable amplitude appear and tags should be properly pre-selected to provide homogeneous backscattering behavior [16]). Besides, a number of techniques exist that exploit the phase variations of the tag backscattered signal (phase difference of arrival, PDOA), taking advantage of the in-phase and quadrature (I-Q) demodulated components of the base-band signal available at the reader coherent receiver [17]-[22]. In [23], the direction-of-arrival (DOA) method is applied to the conveyor belt scenario. By using a two-element array antenna, the direction of arrival of the tag backscattered signal is estimated through a proper phase processing. If multiple readings are available, the tag position estimation can be improved through a least-square fitting processing. In [24], a method exploiting the phase spatial gradient has been proposed to locate moving tags along a known unidirectional path. By accurately detecting the zero-crossing of the average phase gradient, the tag passage at an assigned position is determined.

A more recent knowledge-based method to locate UHF-RFID tags on a conveyor belt has been firstly proposed in [25]-[27], and later on applied in [28]. The basic idea is to simplify localization and tracking of moving tags by using information known $a$ priori (i.e. the conveyor belt path and speed), by adopting a knowledge-based signal processing approach in conjunction with the SAR/ISAR (synthetic aperture radar/inverse SAR) concept [29]. Preliminary results on the localization capability of the proposed method have been illustrated in [30]-[31]. Differently from localization problems in a 3D scenario, it does not need for a set of reader antennas, or synchronized readers, or special radar-like readers with variable interrogation frequency (FMCW, Frequency Modulated Continuous-Wave) [32].

In this paper, the phase-based technique proposed in [25]-[27] stemming from SAR concepts [29], [33]-[34] is described in Section II, with adequate mathematical details and for an arbitrary belt path. In Section III, the performance analysis of such localization method is carried out in terms of location resolution and accuracy, with respect to the main geometrical and system parameters, also producing some guidelines for the system design. Then, in Section IV, some test-case measurements are illustrated to verify the effectiveness of the proposed technique in real scenarios. Finally, conclusions are drawn in Section V.

\section{The Synthetic-Array Phase-Based Tag Localization TeChnique}

Let us consider a conveyor belt lying on the $x y$ plane, and exhibiting an arbitrary path (Fig. 1). Tag positions on the belt follow the curvilinear abscissa $s$, which is aligned with the motion direction. Without any loss of generality, the origin of the spatial coordinate $(s=0)$ is assumed to be coincident with the belt point, along $s$, at minimum distance $r_{M}$ from the reader antenna. The following geometrical and system parameters are considered: RFID operating frequency, $f_{0}$, belt speed, $v$, and interrogation repetition time, IRT. The instantaneous belt speed can be extracted from any control signal at the electrical engine, or measured through a kinetic sensor at the belt border. The reader can detect the tag when the latter is located within the antenna beam (halfpower beamwidth, $H P B W) ;(r, \theta)$ denote the radial and angular tag polar coordinates with respect to the reader antenna.

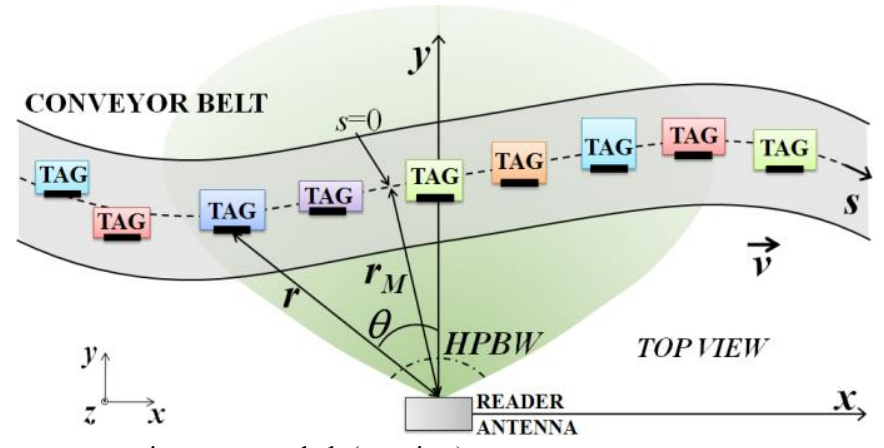

Fig. 1. An RFID scenario with tagged items on a moving conveyor belt (top view).

Thanks to the anti-collision algorithms implemented in UHF-RFID protocols, and also considering that each backscattered signal contains the tag unique identifier, the proposed localization algorithm can be described by assuming that there is only one tag on the belt. Nevertheless, it is worth noting that parallel processing of multiple tags can be easily implemented, so allowing for a real-time localization of all the tags that a conventional wide-beam reader antenna can identify simultaneously, without requiring a one at a time approach.

The reader interrogates the tag several times during its movement along the conveyor belt, with an interrogation repetition time $I R T$, from which a spatial sampling interval $\Delta s=v \cdot I R T$ follows. The number of available readings (successful interrogations), $N_{r}$, is limited by a maximum value that depends on: the distance between reader antenna and belt, the antenna beam and the above 
spatial sampling interval. For simplicity's sake, a uniform sampling is considered throughout Sect. II and Sect. III for the analytical description of the proposed technique, namely the temporal interval IRT is assumed to be constant during the acquisition of the $N_{r}$ readings. Actually, since the reader has to manage all the tags that at the same time are inside the antenna beam, the phase samples for each tag are not regularly distributed across the observation temporal interval. Nonetheless, it is worth to underline that the proposed technique can still be applied, by simply making use of the time stamp of each reading (which is made available as an output of any commercial UHF-RFID reader), and this is what has been done in the measured data analysis (Sect. IV). Moreover, $N_{r}$ can be lower than what is expected in a given observation interval, since some readings can be missed due to multipath and shadowing phenomena, as well as nulls in tag antenna radiation pattern. All these adverse effects also contribute to a non uniform distribution of the phase samples.

It is worth noting that the $N_{r}$ successful readings result in $N_{r}$ complex I-Q samples obtained at the reader side, after coherent baseband demodulation. As typical in RFID signal processing [17], the DC component of the I-Q samples can be filtered out (at each reading), to cancel out the effect of reader transmit-receive leakage and environmental clutter. After this operation, the average value of tag complex backscattering cross section (i.e. the tag static backscatter component) is also compensated for. As a result, the filtered signal is now related to the modulated component only, corresponding to the differential backscattering cross section (the tag communication signal constellation in the phasor I-Q plane resulting to be antipodal). From this, modern RFID chipsets output an RSSI value and importantly a phase delay measure, which can be coded as a complex signal sample.

When the tag moves along the conveyor path, multiple replies are activated by the reader interrogations and the history of the propagation phase-delay due to the two-way reader antenna-tag path can be calculated as:

$$
\varphi_{P_{n}}=\varphi_{0}+4 \pi r_{n} / \lambda, \quad n=1, \ldots, N_{r},
$$

where $\lambda$ is the free-space wavelength of the radiated field and $\varphi_{0}$ is the phase offset including the effect of cables and other reader components [17]. In (1), $r_{n}$ denotes the sequence of relative distance values that changes from reading to reading:

$$
r_{n}=f\left(s_{n}\right) \text { with } s_{n}=g\left(n, s_{0}\right), \quad n=1, \ldots, N_{r} .
$$

In (2), $f(\cdot)$ is a function relating the tag spatial coordinate along the belt, $s_{n}$, to the distance between tag and reader antenna, $r_{n}$. Moreover, $g\left(n, s_{0}\right)$ is the function describing the spatial sampling of the possible tag locations along the belt path, which depends on the hypothesized tag spatial coordinate at a reference time, $s_{0}$. For a given constant belt speed and a set of reading indexes $s$ :

$$
s_{n}=g\left(n, s_{0}\right)=s_{0}+(n-1) v \cdot I R T=s_{0}+(n-1) \Delta s .
$$

However, only the variations of the phase history with respect to the propagation phase at the reference time (i.e. the relative phase history) are actually effective for the proposed analysis:

$$
\Delta \varphi_{P n}=\varphi_{P_{n}}-\varphi_{P_{1}}, \quad n=1, \ldots, N_{r} .
$$

Thus, the term $\varphi_{0}$ is filtered out.

It is important to underline at this point that the relative propagation phase history in (4) is characteristic of the position of the tag at a reference reading time (without loss of generality, the time of the first reading, $n=1$, will be assumed as the reference time in the following, and the corresponding tag position is denoted by $s_{0}$ ). From (4), a phasor data sequence can be assembled:

$$
\boldsymbol{y}_{p}=\left[1, \exp \left(-j \Delta \varphi_{P 2}\right), \cdots, \exp \left(-j \Delta \varphi_{P_{r}}\right)\right]^{T},
$$

with ' $T$ ' being the transpose operator. For a given path, the propagation phasor data sequence, $\boldsymbol{y}_{p}$, coming out from the measured signal samples, varies as a function of $s_{0}$, namely the tag position at the first useful reading. For known belt path and speed, a set of nominal relative phase histories can be computed analytically, for a set of hypothesized values of the tag spatial coordinate $s_{0}$ (denoted as $s_{0}^{\prime}$ in the following):

$$
\mathbf{a}\left(s_{0}{ }^{\prime}\right)=\left[1, \exp \left(-j \Delta \varphi_{P 2}\right), \cdots, \exp \left(-j \Delta \varphi_{P N_{r}}\right)\right]^{T},
$$

with the dependence on $s_{0}{ }^{\prime}$ being through:

$$
\Delta \varphi_{P n}=4 \pi\left(r_{n}-r_{1}\right) / \lambda \text { with } r_{n}=f\left(s_{n}\right) \text { and } s_{n}=g\left(n, s_{0}{ }^{\prime}\right) \text {. }
$$

The best match between the nominal propagation phasor sequence (6) and the propagation phasor data sequence (5), which is used to get an estimate $s_{0}$ of the tag spatial coordinate $s_{0}$ at the reference time, can be found through the similarity measure operator given by a normalized cross-correlation in the spatial domain [35]:

$$
s_{0}=\arg \max _{s_{0}{ }^{\prime}} \frac{\left|\boldsymbol{a}^{\boldsymbol{H}}\left(s_{0}{ }^{\prime}\right) \boldsymbol{y}_{\boldsymbol{p}}\right|^{2}}{\left\|\boldsymbol{a}^{\boldsymbol{H}}\left(s_{0}{ }^{\prime}\right)\right\|^{2} \cdot\left\|\boldsymbol{y}_{\boldsymbol{p}}\right\|^{2}},
$$

with ' $H$ ' being the Hermitian (conjugate transpose) operator and ' $\|$ ' ' the norm operator. The fitting maximization in (8) can be carried out over the grid of $s 0^{\prime}$ values for which the nominal phase history has been computed.

It is worth underlying that the relative motion between the tag and the reader antenna due to the conveyor belt movement can also be considered as produced by a virtual motion of the reader antenna with respect to the tag virtually considered to be steady, 
and this results in a synthetic array being produced, so resembling a SAR approach [29]. In this context, the nominal propagation phasor sequence (6) codes the response of the synthetic array to a backscattering originating from a scatterer displaced along the belt by $s_{0}^{\prime}$ with respect to the reader antenna position. The latter response can be termed as steering vector [33]-[34], since it can be exploited to estimate the backscattering coordinate with a matching process physically equivalent to form and steer a fine virtual beam from the synthetic array, until the backscattering source is detected. Then, the fitting maximization in (8) basically corresponds to searching for the steering vector onto which the projection of the propagation phasor data sequence obtained from the data is maximum.

As an example, a set of simulated relative phase histories (4) are shown in Fig. 2, together with the outputs of the matching operator in (8), with reference to a rectilinear path. The following geometrical and system parameters have been used to get the results in Fig. 2: $f_{0}=866 \mathrm{MHz}, r_{M}=2 \mathrm{~m}, v=1 \mathrm{~m} / \mathrm{s}, I R T=100 \mathrm{~ms}$, and $\Delta s=10 \mathrm{~cm}$. The relative phase histories are represented as a function of the spatial coordinate $s_{n}$, for three tags that appear at different positions at the reference time $\left(s_{O A}=-1.1 \mathrm{~m}, s_{O B}=-0.8 \mathrm{~m}\right.$ and $s_{O C}=-0.4 \mathrm{~m}$ ). Within the reader antenna beam, an equal number of available readings has been considered for all the tags: $N_{r}=16$. A sizeable difference between the three curves is apparent; in particular, different portions of the phase history are observed depending on the tag position at the reference time, so showing that a propagation phase history analysis can be profitably used to identify the tag location at an assumed reference time. From the matching operator output in Fig. 2, the curve peak position corresponds to the hypothetical tag position $s_{0}{ }^{\prime}$ associated to the nominal phase history that best fits the relative phase history (4); the peak position is chosen as the most likely position of the tag at the reference time, $s_{0}$, as from eq. (8).

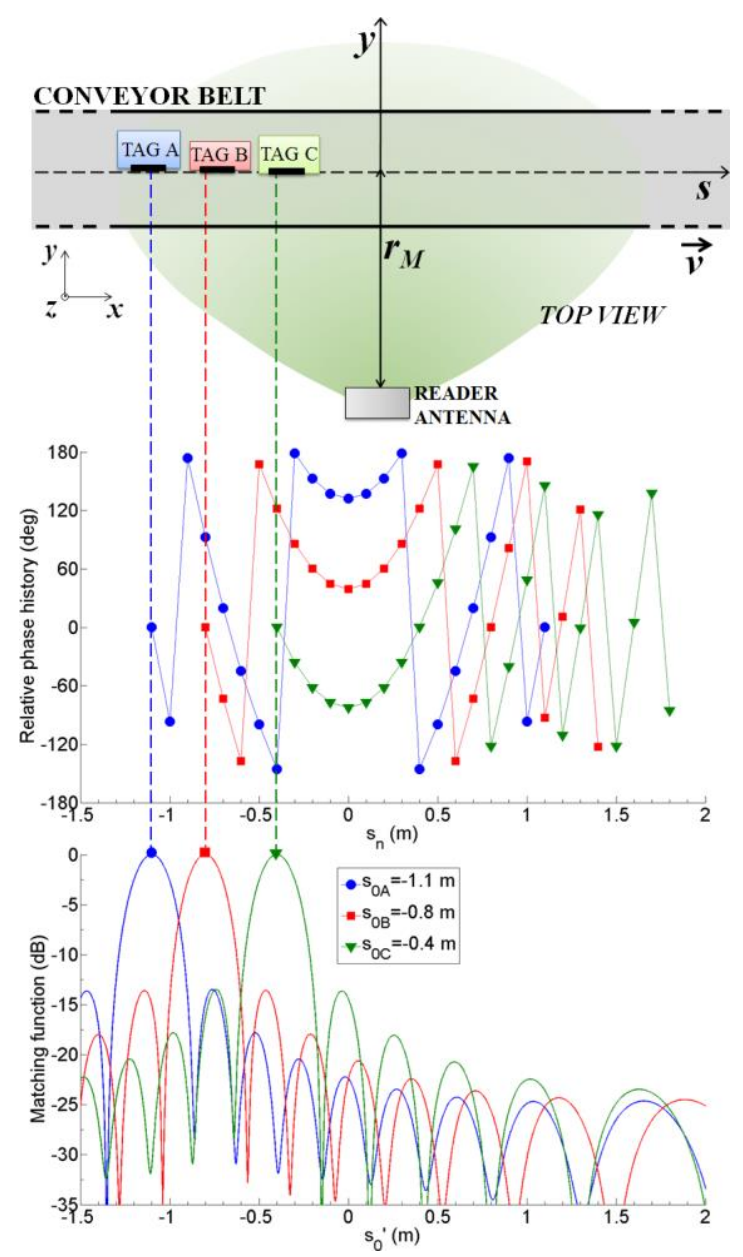

Fig. 2. A set of relative phase histories of the complex signals at the reader receiver versus the spatial coordinate $s_{n}$, and the corresponding matching operator outputs versus the hypothetical tag position at the reference time $s o^{\prime}$. Results are obtained for three tags occupying different positions at the reference time (time of the first useful reading): $s_{O A}=-1.1 \mathrm{~m}$ (circle blue markers), $s_{O B}=-0.8 \mathrm{~m}$ (square red markers) and $s_{O C}=-0.4 \mathrm{~m}$ (triangle green markers). Curves are relevant to a rectilinear path. An equal number of available readings has been considered for all the tags, $N_{r}=16$; other parameters: $f_{0}=866 \mathrm{MHz}, r_{M}=2 \mathrm{~m}, v=1 \mathrm{~m} / \mathrm{s}$, $I R T=100 \mathrm{~ms}$ and $\Delta s=10 \mathrm{~cm}$. 


\section{PERFORMANCE ANALYSIS}

The effect of the main system parameters on the tag localization accuracy is firstly analyzed for a rectilinear path, and then extended to a semi-circular path.

\section{A. System resolution and accuracy}

In Fig. 3, examples of the normalized matching function output are given, with respect to the hypothetical tag position $s_{0}$ at the reference time (time of the first reading, $n=1$ ), when the tag moves on a rectilinear path. It has been assumed that the actual tag position at the reference time is $s_{0}=-1.1 \mathrm{~m}$ (location of tag A in Fig. 2) and the geometrical and system parameters are the same as those used to get the numerical results in Fig. 2. Different curves are associated to different values of the extension of the spatial interval $D=\Delta s\left(N_{r}-1\right)$ in which the tag is detected by the reader: $D=1,1.5$ and $2 \mathrm{~m}$ ( $D$ is also the aperture of the synthetic array, if the SAR analogy is considered). The observation spatial interval always begins at $s_{0}=-1.1 \mathrm{~m}$. It has been assumed that $D$ changes because the number of the processed samples of the relative phase history $N_{r}$ changes, while the spatial sampling interval $\Delta s$ remains constant. The observation time interval $T$ needed to collect $N_{r}$ readings can be derived as: $T=D / v=I R T\left(N_{r}-1\right)$.

In all the case studies of Fig. 3, the presence of a well distinct peak and a low level of the secondary lobes confirm the good localization capabilities of the matching method (or equivalently, the fine focusing around the true tag position of the received signal power at the synthetic array). From the point associated to the matching function peak, the tag position at the reference time $s_{0}$ is estimated. Obviously, a theoretical infinite precision is obtained in an ideal scenario (namely $s_{0}$ always coincides with $s_{0}$ in the numerical simulations). Nonetheless, a narrow width of the matching function main lobe (i.e. a good algorithm resolution) indicates that good performance can be obtained also in presence of localization error sources, such us multipath, environmental clutter and thermal noise. Thus, it is important to carry out a basic localization capability investigation through the analysis of the system resolution in ideal conditions. In fact, the localization precision is not the resolution level, yet for typical signal-to-noise ratios the precision in array processing is finer than the resolution [36], as it has been confirmed by the measurement campaign results.

If the monolateral width at the first null position of the matching function mainlobe, $R_{s}$, is assumed as the system resolution, the spatial broadside resolution of a rectilinear synthetic array can be calculated as [33]:

$$
R=r_{M} \frac{\lambda}{2 D} .
$$

For the cases in Fig. 3, the resolution extracted from the numerical simulations is equal to: $R_{s}=34,23$ and $17 \mathrm{~cm}$, when $D=1,1.5$ and $2 \mathrm{~m}$, respectively. Above values differ of less than $2 \%$ from those predicted by eq. (9). Such a small difference was expected as the assumptions used to derive eq. (9) are not met when the scatterer is in the antenna near-field region [33]. Nevertheless eq. (9) represents an useful formula to estimate the expected resolution level. Eq. (9) and numerical results show that a finer spatial resolution can be obtained if the observation spatial interval increases (even if a longer data acquisition time, $T$, has to be tolerated).

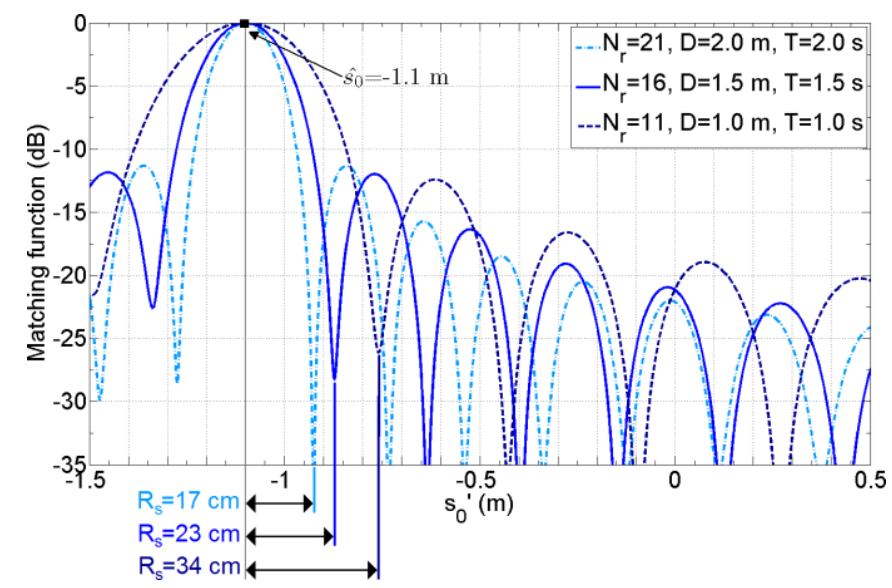

Fig. 3. Matching function versus the hypothetical tag position $s o^{\prime}$ (actual tag position at the reference time is $s 0=-1.1 \mathrm{~m}$ ) for different observation spatial intervals $D$, which are relevant to a set of number of processed readings: $D=2 \mathrm{~m}$ and $N_{r}=21$ (dash-dotted line), $D=1.5 \mathrm{~m}$ and $N_{r}=16$ (solid line), $D=1 \mathrm{~m}$ and $N_{r}=11$ (dashed line). $T$ represents the extension of the time interval in which the tag is detected by the reader and $R_{s}$ is the spatial resolution (monolateral width at the first null position of the matching function mainlobe).

In Fig. 4, a contour plot of the system resolution $R_{s}$ (quantified from the simulated results, as in Fig. 3) has been represented as a function of both the minimum distance between the belt and the reader antenna, $r_{M}$, and the observation spatial interval $D$ (synthetic aperture). As also predicted by eq. (9), the same system resolution can be achieved for several $\left(r_{M}, D\right)$ pairs. Then, the 
proper pair can be chosen as a tradeoff between the room available around the belt and the maximum tolerable observation interval $D$.

In the above resolution chart, curves related to the minimum reader antenna beamwidth, $H P B W_{\min }$, required to collect data within the synthetic aperture $D$ for a given reader antenna-belt distance $r_{M}$, have also been added: $H P B W_{\min }=2 \operatorname{arctg}\left(D / 2 r_{M}\right)$. Those curves are useful to find the $H P B W_{\min }$ required to obtain the wished system spatial resolution. It is also worth noting that the $H P B W_{\min }$ curves (dashed lines) are nearly aligned with the constant-resolution curves (continuous level curves). This basically means that, for a given operating frequency, the physical antenna beam determines the achievable resolution of the syntheticarray based localization system. Such result resembles the resolution limit in SAR systems, where the physical antenna size determines the lower bound of the system resolution [29].

At this point, it has to be noted that, within the spatial interval $D$, the readings have to be collected in basic accordance with the sampling theorem [37], to guarantee the possibility to estimate unambiguously the tag position from its relative phase history (namely, to avoid an excessive level of the secondary lobes in the matching function output). In particular, the spatial sampling interval $\Delta s$ must satisfy the condition $\Delta s \leq \lambda /[4 \sin (H P B W / 2)]$. By considering that $\Delta s=v \cdot I R T$, the criterion to set the interrogation repetition time, IRT, can be derived: $I R T \leq \lambda /[4 v \sin (H P B W / 2)]$. In real applications the spatial sampling is not uniform [38], and it is in principle enough that a couple of spatial samples satisfies the condition above.

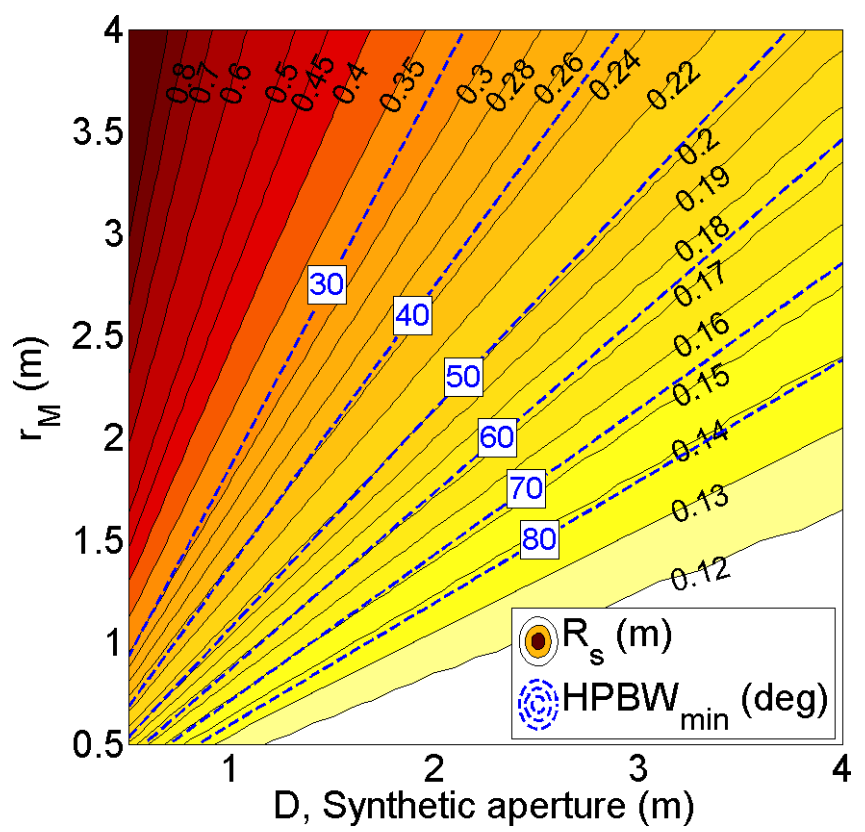

Fig. 4. Contour plot of the system resolution $R_{s}$ with respect to the observation spatial interval $D$ in which the tag is detected by the reader, and the reader antenna-belt distance $r_{M} ; f_{0}=866 \mathrm{MHz}, v=1 \mathrm{~m} / \mathrm{s}, I R T=100 \mathrm{~ms}$. As a reference, curves of the minimum reader antenna beam width $H P B W_{\min }$ required to collect data within the synthetic aperture $D$ for a given reader antenna-belt distance, have also been added (dashed lines).

\section{B. Semi-circular conveyor belt}

Further numerical results are given for a semi-circular path lying on the $x y$ plane (Fig. 5), to show the localization technique capabilities for not-rectilinear conveyors. The reader antenna is displaced from the circle center, and internally (or externally) located with respect to the conveyor belt circle. $r_{M}$ still denotes the minimum distance between the reader antenna and the belt.

As an example, the matching operator output is shown in Fig. 6, for a radius of curvature of the belt path equal to $r_{b p}=4 \mathrm{~m}$, when the following parameters are employed: $f_{0}=866 \mathrm{MHz}, r_{M}=2 \mathrm{~m}, v=1 \mathrm{~m} / \mathrm{s}, I R T=100 \mathrm{~ms}$. It has been assumed that tag position at the reference time is $s_{0}=-1 \mathrm{~m}$, with an extension of the observation interval along the curvilinear path equal to $D=2 \mathrm{~m}$, and $N_{r}=21$. A well distinct peak is apparent also in this case, with a relatively low level of the secondary lobes. Let us make a comparison against the resolution relevant to the rectilinear path case, for which the same reader antenna-belt minimum distance $\left(r_{M}=2 \mathrm{~m}\right)$ and available readings number $\left(N_{r}=21\right)$ have been considered. When the reader antenna is located inside the conveyor belt circle, the system resolution is coarser $\left(R_{s}=34 \mathrm{~cm}\right.$ instead of the $R_{s}=17 \mathrm{~cm}$ in Fig. 3), as expected for the more smooth variations of the phase history (due to the reduced variations in the reader antenna-tag distance along the belt). In Fig. 6, the results for the case of a reader antenna placed outside the conveyor belt circle show how more significant variations of the phase history can lead to a resolution finer than that for a rectilinear path: $R_{s}=11 \mathrm{~cm}$. 


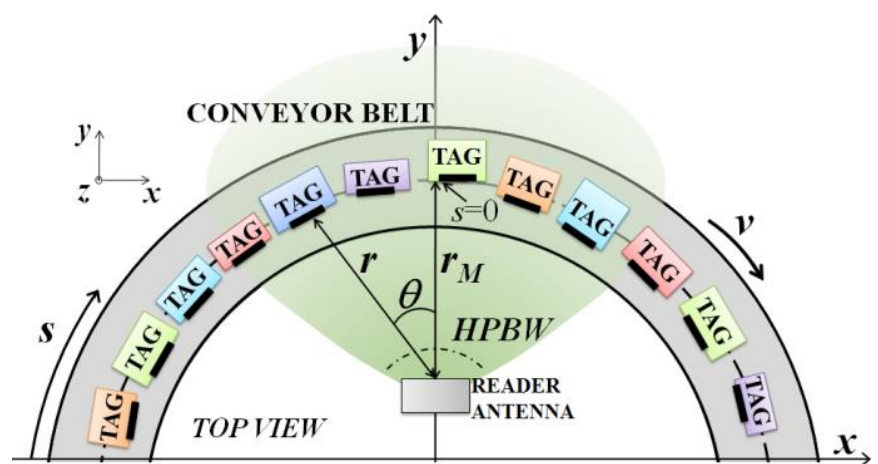

Fig. 5. An RFID scenario with tagged items on a semi-circular conveyor belt (top view). The antenna can be either internally (as in the figure) or externally located with respect to the belt circle.

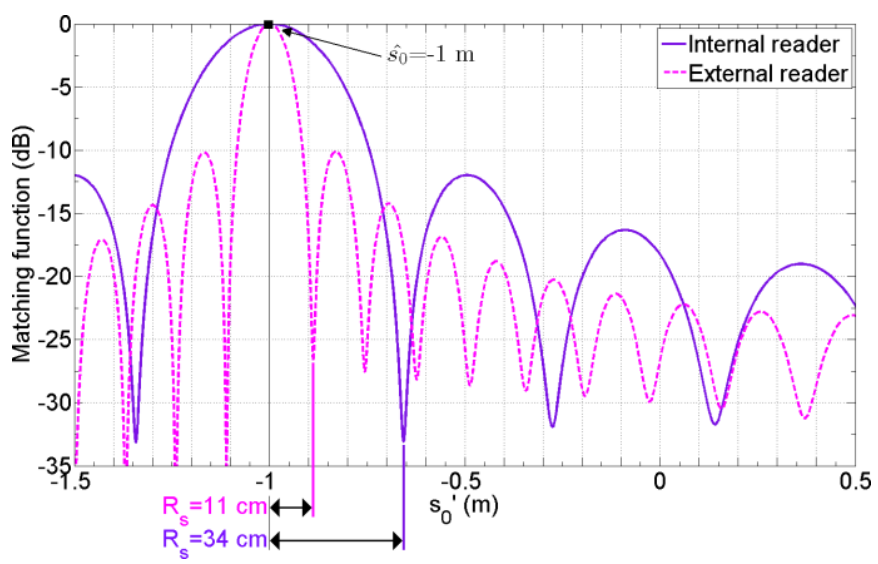

Fig. 6. Matching function versus the hypothetical tag position $s o^{\prime}$, for the curvilinear conveyor belt $\left(r_{b p}=4 \mathrm{~m}\right.$ ), when the reader is internal (solid line) or external (dashed line) with respect to the conveyor circle. The actual tag position at the reference time is $s_{0}=-1 \mathrm{~m}, D=2 \mathrm{~m}$ and the available readings number is $N_{r}=21$. Other parameters: $f_{0}=866 \mathrm{MHz}, r_{M}=2 \mathrm{~m}, v=1 \mathrm{~m} / \mathrm{s}, I R T=100 \mathrm{~ms}$.

\section{Algorithm LocAlization CAPABILITIES IN A REAL SCENARiO}

In indoor environments, the multipath phenomena represent the main localization error source [39]. The effect of multiple waves reaching the reader after reflections at walls and floor surfaces can be efficiently studied by resorting to deterministic propagation models [40], as a function of the system parameters and scenario geometry. However, a measurement campaign in real scenarios allows to account for additional phenomena, such as thermal noise, environmental clutter, tag rotation during phase data acquisition, etc. In such framework, an extensive measurement campaign has been carried out at a student restaurant of the University of Pisa, where a rectilinear conveyor belt is available for trays recovery (a few preliminary results have been presented in [30]). The measurement setup is shown in Fig. 7. The commercial reader Intermec IF2 has been employed, which is able to measure the phase of the tag backscattered complex signal with 1 degree precision. Two different circularly polarized antennas have been used: a) a WANTENNAX005 CAEN antenna, with $H P B W_{H}=H P B W_{V}=67^{\circ}$ and Gain=6.5 dBic; b) a MT$243017 / \mathrm{NRH} / \mathrm{B}$ MTI Wireless Edge antenna, with $H P B W_{H}=62^{\circ}, H P B W_{V}=30^{\circ}$ and Gain $=12 \mathrm{dBic}$. The reader output power and frequency channel have been set to $P_{\text {OUT }}=200 \mathrm{~mW}$ and $f_{0}=866.2 \mathrm{MHz}$ (ETSI channel 4), respectively. On the basis of what has been remarked about the non regular distribution of measured phase samples in the observation temporal interval $T$ (see Sect. II), in this section the parameter IRT has to be intended as an average value of the inter-sample distance in the time-series phase data. 


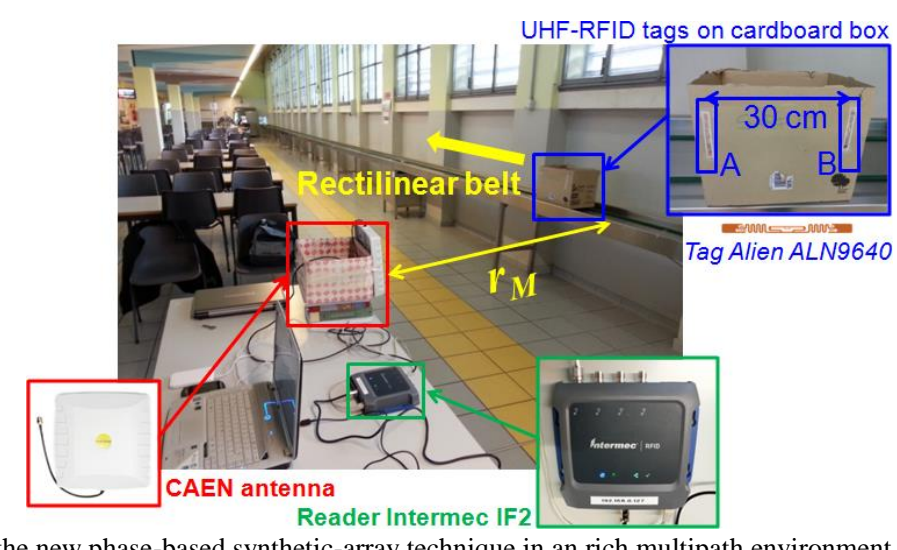

Fig. 7. Measurement setup to validate the new phase-based synthetic-array technique in an rich multipath environment.

To verify both sorting and localization capabilities of the proposed phase-based technique, two UHF RFID tags, (ALN9640 squiggle inlay tags) have been placed at a distance $d_{A B}=30 \mathrm{~cm}$ on a cardboard box moving on the conveyor belt (Tag A and Tag B in Fig. 7). An example of the measured phase histories is shown in Fig. 8a (system parameters: $r_{M}=2.1 \mathrm{~m}, v=0.1 \mathrm{~m} / \mathrm{s}$, $I R T=100 \mathrm{~ms}$, MTI Wireless Edge antenna). $N_{r, A}=176$ and $N_{r, B}=178$ readings are collected for tags A and B, respectively. The multipath effect is apparent from the non-smooth behavior of the phase curves. Nonetheless, the proposed localization technique is robust with respect to such phenomena, as apparent from the matching function in Fig. 8b. Indeed, two well distinct peaks appear, with a low level of the secondary lobes. The estimated position of the two tags, at the time of the first reading, is $s_{0, A}=-1.039 \mathrm{~m}$ and $s_{0, B}=-1.347 \mathrm{~m}$ for tags A and B, respectively. The sorting is done correctly and the estimated inter-tags distance is $d_{A B}=30.8 \mathrm{~cm}$, with an absolute error of only $0.8 \mathrm{~cm}$. The measured system resolutions are $R_{s, A}=18.1 \mathrm{~cm}$ and $R_{s, B}=18.6 \mathrm{~cm}$, which are in good agreement with the value of $R=19.4 \mathrm{~cm}$ predicted from eq. (9) for $D=1.8 \mathrm{~m}$ and $r_{M}=2.1 \mathrm{~m}$.

To evaluate the localization accuracy, namely the error of the estimated tag position, it is necessary to know the actual tag position at the reference time. During measurements, the reader query command needed to start a continuous inventory was sent when the first tag was crossing the known location at $s_{0}=-1.05 \mathrm{~m}$ (which has been selected to be within the reader antenna $H P B W$ ). Thus, such coordinate represents the reference position with respect to which the localization error has to be calculated for the forward tag, $s_{0, A}=-1.05 \mathrm{~m}\left(s_{0, B}=-1.35 \mathrm{~m}\right.$ has been then assumed as the position of the backside tag, as tags $\mathrm{A}$ and $\mathrm{B}$ are $30 \mathrm{~cm}$ separated). By assuming the above spatial reference, the absolute localization errors are evaluated as $2.1 \mathrm{~cm}$ and $0.3 \mathrm{~cm}$, for tags A and B, respectively. 


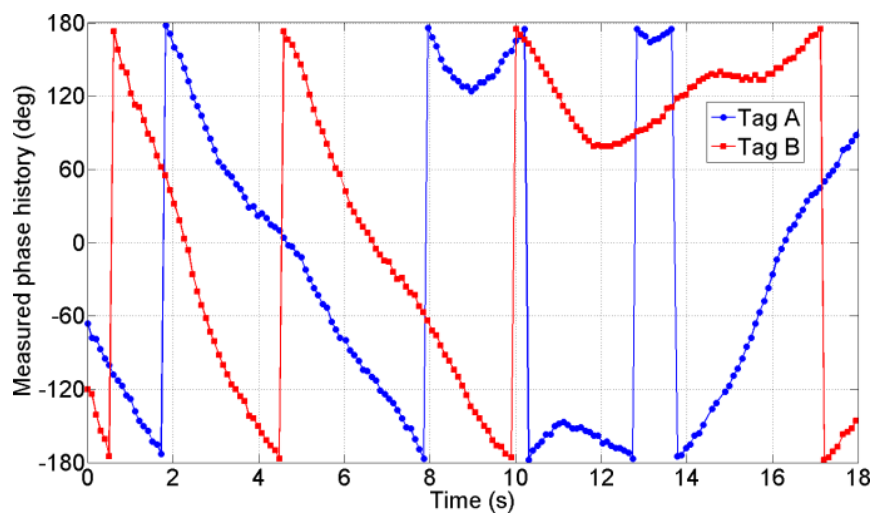

(a)

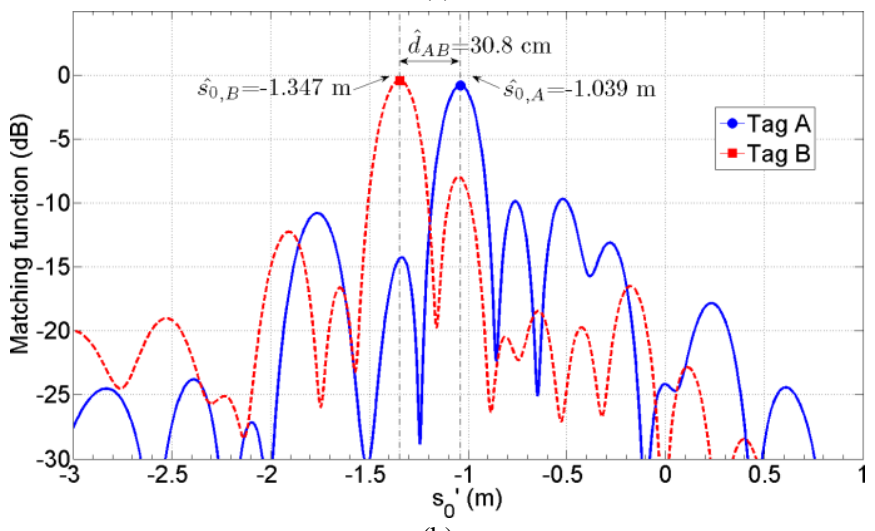

(b)

Fig. 8. (a) Measured phase history at the reader versus time and (b) matching function versus the hypothetical tag position $s 0^{\prime}$, for two tagged items moving on the conveyor belt. The MTI Wireless Edge antenna has been employed and the system parameters are the following: $r_{M}=2.1 \mathrm{~m}, \nu=0.1 \mathrm{~m} / \mathrm{s}, I R T=100 \mathrm{~ms}$, $N_{r, A}=176$ and $N_{r, B}=178$ for tag A and B, respectively.

Localization capability has been verified by performing 7 repeated tests with nearly the same synthetic aperture $D=1.8 \mathrm{~m}$, and statistics from measured results are illustrated in Table $\mathrm{I}$ in terms of bias and standard deviation values. The sorting is always done correctly, and the statistics on the estimated distance among tags are: [bias $\left.\left(d_{A B}\right), \sigma\left(d_{A B}\right)\right]=[2.3 \mathrm{~cm}, 1.8 \mathrm{~cm}]$. Such results confirm the good sorting capability, with centimeter order accuracy in the estimated distance among tags. The statistics on the estimated absolute tag position are calculated by averaging results related to both tags (14 test cases): [bias ( $\left.s_{0}\right)$, $\left.\sigma\left(s_{0}\right)\right]=[6.4 \mathrm{~cm}, 3.2 \mathrm{~cm}]$. Apparently, contingent synchronization errors between the time on which the tag passes at the known position and that on which the reader query command is sent determine an error in the calculation of $s_{0}$, which has been used here as a reference. However, due to the relatively low speed of the conveyor, it is expected that such error is not much influential to the localization performance evaluation. Statistics on the measured resolution are included in Table I, by considering the value predicted from eq. (9) as a reference value for the expected resolution: $R=19.4 \mathrm{~cm}$, when $r_{M}=2.1 \mathrm{~m}, D=1.8 \mathrm{~m}$ and $f_{0}=866.2 \mathrm{MHz}$. Both the bias of the measured resolution with respect to the predicted one and its standard deviation are close to $1 \mathrm{~cm}$. Thus, the measurement campaign confirms that the theoretical resolution given from eq. (9) represents an useful formula to evaluate the expected resolution in real scenarios with rectilinear conveyor belts.

\section{A. Effect of synthetic aperture size $D$ and belt speed $v$}

In the test results shown above, the phase-based localization technique has been applied after collecting all data samples in a spatial observation interval $D=180 \mathrm{~cm}$, while tags were moving along the conveyor belt. However, the localization algorithm can be executed as soon as new phase samples are measured. Statistics are illustrated in Table I by processing a different number of data samples acquired for the above 7 test cases (14 test cases for the estimated tag position): $D=80,130$ and $180 \mathrm{~cm}$. The statistics on the estimated distance and position are of centimeter order, for all considered values of $D$. Moreover, the longer is the synthetic array, the finer is the system precision (the bias and generally also the standard deviation decrease). This suggests that after collecting some readings, in real time, the localization technique can be applied; after that, the result can be improved as soon as new data are collected. The statistics on the system resolution confirm that the theoretical value predicted from eq. (9) is a good approximation $\left(R=26.9 \mathrm{~cm}\right.$ with $D=130 \mathrm{~cm}$ and $r_{M}=2.1 \mathrm{~m} ; R=43.7 \mathrm{~cm}$ with $D=80 \mathrm{~cm}$ and $\left.r_{M}=2.1 \mathrm{~m}\right)$; for the smallest synthetic aperture value, a poorer approximation is obtained: bias $\left(R_{s}\right)=5 \mathrm{~cm}$ for $D=80 \mathrm{~cm}$. 
The effect of the belt speed has also been considered, as the latter is an important parameter to improve the efficiency of the goods management systems. If the speed increases, less phase samples will be collected within the synthetic aperture $D$. In our tests, this effect has been simulated by undersampling the phase history collected with the actual belt speed $(v=0.1 \mathrm{~m} / \mathrm{s})$, which is equivalent to consider a longer interrogation repetition time IRT. Thus, measurement data have been re-processed by assuming $I R T=500 \mathrm{~ms}$ (emulating the case with $v=0.5 \mathrm{~m} / \mathrm{s}$ ) and $I R T=1000 \mathrm{~ms}$ (emulating the case with $v=1 \mathrm{~m} / \mathrm{s}$ ), with the same synthetic aperture $D=180 \mathrm{~cm}$ for both cases. Statistics in Table II show that the algorithm performance is quite stable and the localization capability is maintained. This result was expected since for all cases the measured phase samples are collected in accordance with the criterion $I R T \leq \lambda /[4 v \sin (H P B W / 2)]$. In Table II, $N_{r}$ represents the average number of available readings among the considered repeated tests.

TABLE I

STATISTICS ON THE ESTIMATED DISTANCE AMONG TAGS, THE ESTIMATED TAG POSITION AND THE MEASURED RESOLUTION, VS. THE SYNTHETIC APERTURE $D$ (SYSTEM PARAMETERS: $r_{M}=2.1 \mathrm{~m}, I R T=100 \mathrm{~ms}, v=0.1 \mathrm{~m} / \mathrm{s}$, MTI Wireless Edge antenna).

\begin{tabular}{c|c|c|c}
\hline \hline Synthetic aperture, $D$ & $80 \mathrm{~cm}$ & $130 \mathrm{~cm}$ & $180 \mathrm{~cm}$ \\
\hline \hline & {$[$ bias, $\sigma] \mathbf{c m}$} & {$[$ bias, $\sigma] \mathbf{c m}$} & {$[$ bias, $\sigma] \mathbf{c m}$} \\
\hline \hline$d_{A B}$ & {$[7.4,6.1]$} & {$[3.7,2.2]$} & {$[2.3,1.8]$} \\
\hline$s_{0}$ & {$[7.3,4.2]$} & {$[5.3,3.3]$} & {$[6.4,3.2]$} \\
\hline$R_{s}$ & {$[5.0,5.4]$} & {$[2.4,5.3]$} & {$[0.7,0.6]$} \\
\hline
\end{tabular}

TABLE II

STATISTICS ON THE ESTIMATED DISTANCE AMONG TAGS, THE ESTIMATED TAG POSITION AND THE MEASURED RESOLUTION, VS. THE IRT (SYSTEM PARAMETERS: $r_{M}=2.1 \mathrm{~m}, v=0.1 \mathrm{~m} / \mathrm{s}, D=180 \mathrm{~cm}$, MTI Wireless Edge antenna).

\begin{tabular}{c|c|c|c}
\hline \hline$I R T$ & $100 \mathrm{~ms}$ & $500 \mathrm{~ms}$ & $1000 \mathrm{~ms}$ \\
\hline \hline$N_{r}$ & 174 & 36 & 19 \\
\hline \hline & {$[$ bias, $\sigma] \mathrm{cm}$} & {$[$ bias, $\sigma] c m$} & {$[$ bias, $\sigma] \mathbf{c m}$} \\
\hline$d_{A B}$ & {$[2.3,1.8]$} & {$[2.3,1.7]$} & {$[2.4,1.6]$} \\
\hline$S_{0}$ & {$[6.4,3.2]$} & {$[6.5,3.2]$} & {$[6.5,3.2]$} \\
\hline$R_{s}$ & {$[0.7,0.6]$} & {$[1.3,0.7]$} & {$[1.7,0.7]$} \\
\hline
\end{tabular}

\section{B. Effect of reader antenna beamwidth and inter-tags distance}

To further verify the robustness of the new localization technique with respect to multipath phenomena, a less directive reader antenna (i.e. a CAEN antenna) has been employed. Indeed, its wider beamwidth on the vertical plane $\left(H P B W_{V}=67^{\circ}\right.$ instead of the $H P B W_{V}=30^{\circ}$ exhibited by the MTI Wireless Edge antenna) is expected to increase the number of reflected rays intercepted within the antenna beam. In Fig. 9a, samples of measured phase histories are given, when $r_{M}=2.05 \mathrm{~m}, v=0.1 \mathrm{~m} / \mathrm{s}, I R T=100 \mathrm{~ms}$ and $D=150 \mathrm{~cm}$. A quite large number of missed readings and an apparent non-uniform sampling indicate the presence of a strong multipath. As a consequence, a higher level of the secondary lobes appears in the matching functions (see Fig. 9b). However such a level is at least $3 \mathrm{~dB}$ below the peak and no ambiguity is introduced in the tag position estimation. The absolute localization errors are $9 \mathrm{~cm}$ and $10.1 \mathrm{~cm}$, for tags $\mathrm{A}$ and $\mathrm{B}$, respectively (the tags assumed positions at the reference time are still as $s_{0, A}=-$ $1.05 \mathrm{~m}$ and $s_{0, B}=-1.35 \mathrm{~m}$ ), and this is one of the worst cases. The achieved system resolution is still close to that one calculated through eq. (9), since the synthetic aperture $D$ is the only significant parameter in this respect, despite of a non-uniform spatial sampling. 


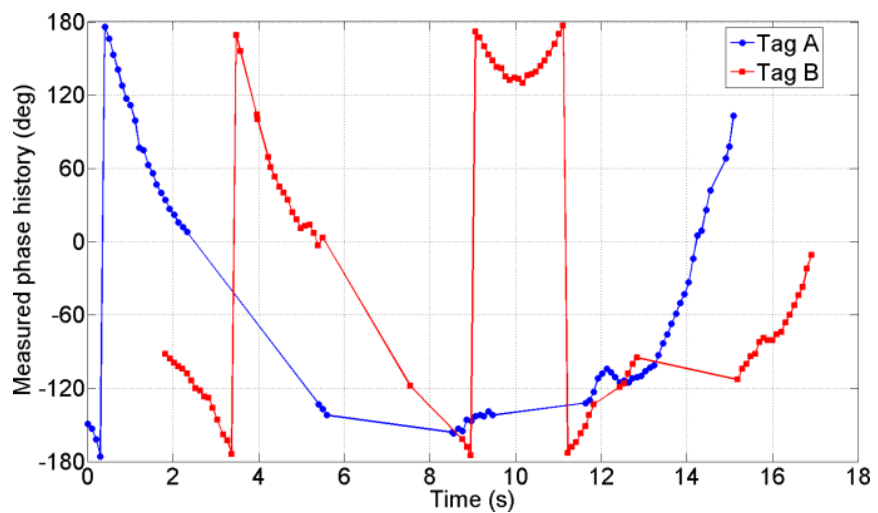

(a)

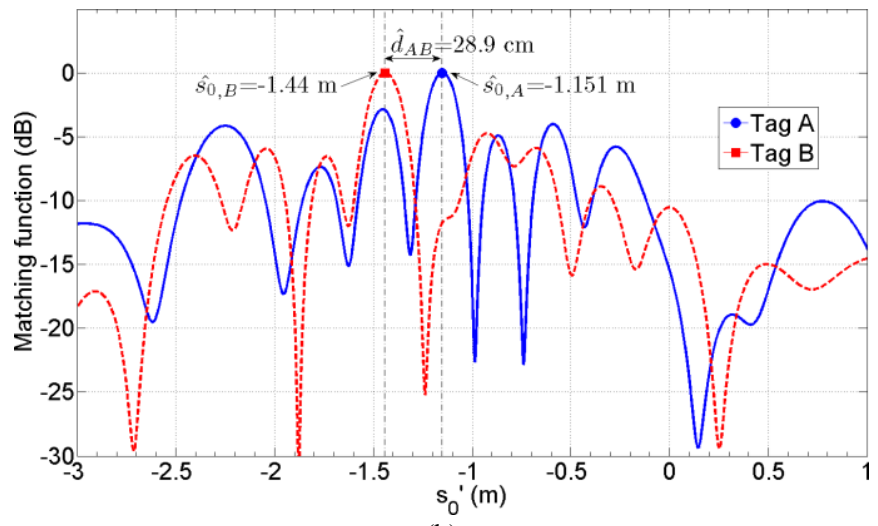

(b)

Fig. 9. (a) Measured phase history at the reader versus time and (b) matching function versus the hypothetical tag position $s 0^{\prime}$, for two tagged items moving on the conveyor belt. The CAEN antenna has been employed and the system parameters are the following: $r_{M}=2.05 \mathrm{~m}, v=0.1 \mathrm{~m} / \mathrm{s}, I R T=100 \mathrm{~ms}, N_{r, A}=92$ and $N_{r, B}=71$ for tag A and B, respectively.

Five test cases have been carried out for each value of the distance among the two tags $\left(d_{A B}=10,20,30,40 \mathrm{~cm}\right)$ to verify the effect of the tag mutual coupling. The following system parameters have been considered: $r_{M}=2.0 \mathrm{~m}, v=0.1 \mathrm{~m} / \mathrm{s}, I R T=100 \mathrm{~ms}$ and $D=150 \mathrm{~cm}$ (CAEN antenna has been used). For all cases, the sorting is done correctly. Statistics are illustrated in Table III, where $N_{r}$ represents the average number of available readings among the considered repeated tests.

A centimeter order accuracy for the estimated distance ( 5 test cases) and the estimated tag position (10 test cases) can be observed independently from the inter-tags distance, except for the challenging case of $d_{A B}=10 \mathrm{~cm}$, when the largest bias in the estimated distance is achieved: bias $\left(d_{A B}\right)=9.3 \mathrm{~cm}$ (average overestimate, with sorting still not affected). This suggests that a minimum inter-tags distance exists, which should be guaranteed to limit some performance degradation related to tag mutual coupling. However, a minimum inter-tags distance $d_{A B} \approx 10 \mathrm{~cm}$ represents and adequate bound for those widespread applications where the tagged items are relatively large (e.g. bags in airport baggage handling systems, boxes in parcel distribution centers). Finally, it is worth noting that the above test cases have been carried out in the worst condition of parallel tags, which determines a stronger mutual coupling.

TABLE III

STATISTICS ON THE ESTIMATED DISTANCE AMONG TAGS, THE ESTIMATED TAG POSITION AND THE MEASURED RESOLUTION, VS. THE DISTANCE AMONG TAGS

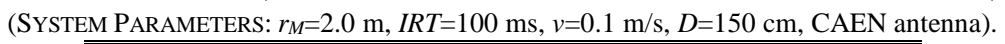

\begin{tabular}{c|c|c|c|c}
\hline \hline$d_{A B}$ & $10 \mathrm{~cm}$ & $20 \mathrm{~cm}$ & $30 \mathrm{~cm}$ & $40 \mathrm{~cm}$ \\
\hline \hline$N_{r}$ & 100 & 130 & 125 & 110 \\
\hline \hline & [bias, $\sigma] \mathbf{c m}$ & [bias, $\sigma] \mathrm{cm}$ & [bias, $\sigma] \mathrm{cm}$ & [bias, $\sigma] \mathrm{cm}$ \\
\hline \hline$d_{A B}$ & {$[9.3,0.6]$} & {$[1.6,0.6]$} & {$[3.5,2.4]$} & {$[3.1,1.9]$} \\
\hline$S_{0}$ & {$[4.7,3.2]$} & {$[5.6,1.0]$} & {$[4.0,3.3]$} & {$[2.5,1.4]$} \\
\hline$R_{s}$ & {$[3.3,2.4]$} & {$[1.4,0.4]$} & {$[1.7,0.9]$} & {$[2.1,2.9]$} \\
\hline
\end{tabular}




\section{Test cases with four moving tags}

Finally, since the phase-based localization technique can work with an arbitrary number of tags moving along the conveyor belt and simultaneously illuminated by the antenna beam, some tests have been carried out by using four tags on the conveyor belt. The tags are at a relative distance of $d=30 \mathrm{~cm}\left(d_{A B}=d_{B C}=d_{C D}=30 \mathrm{~cm}\right)$. A sample of the matching functions is represented in Fig. 10, when the MTI Wireless Edge antenna is used and $r_{M}=1.95 \mathrm{~m}, v=0.1 \mathrm{~m} / \mathrm{s}, I R T=100 \mathrm{~ms}, D=160 \mathrm{~cm}$. The reference position of the first tag at the first reading is $s_{0, \mathrm{~A}}=-0.85 \mathrm{~m}$ (consequently, $s_{0, \mathrm{~B}}=-1.05 \mathrm{~m}, s_{0, \mathrm{C}}=-1.35 \mathrm{~m}$ and $s_{0, \mathrm{D}}=-1.65 \mathrm{~m}$ ).

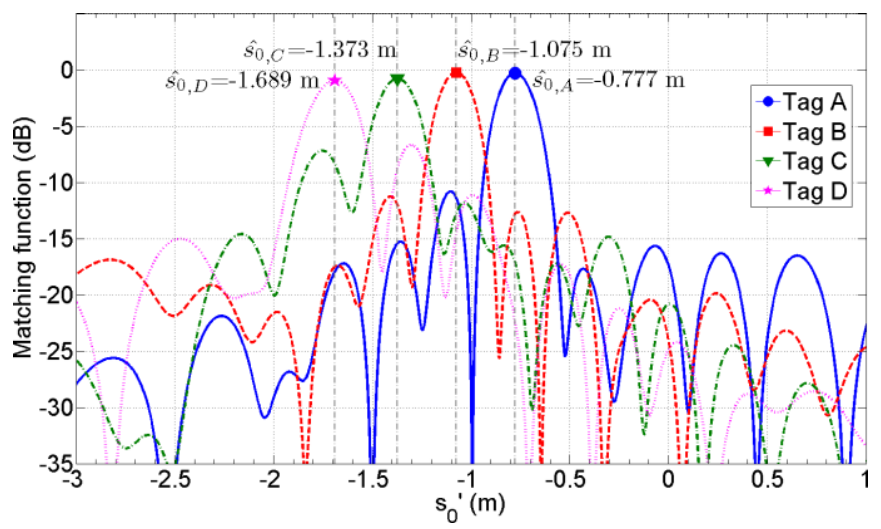

Fig. 10 Matching functions versus the hypothetical tag position $s o^{\prime}$ for four tagged items moving on the conveyor belt. The Wireless Edge MTI antenna has been employed and the following system parameters have been considered: $r M=1.95 \mathrm{~m}, v=0.1 \mathrm{~m} / \mathrm{s}, I R T=100 \mathrm{~ms}$. The synthetic aperture is $D=160 \mathrm{~cm}$ for all tags.

A well distinct peak can be observed for each matching function, together with a low level of the secondary lobes. Statistics have been calculated on 7 repeated tests. Thus, the statistics on the estimated distance are calculated on 21 measurements and the results are as follow: [bias $(d), \sigma(d)]=[3.1 \mathrm{~cm}, 3.3 \mathrm{~cm}]$. The sorting is always correct and a centimeter order accuracy in the estimated distance is maintained. For the estimated tag position, the statistics obtained from the 28 measurements are [bias $\left(s_{0}\right)$, $\left.\sigma\left(s_{0}\right)\right]=[7.8 \mathrm{~cm}, 6.3 \mathrm{~cm}]$.

It is worth noting that such technique is also able to distinguish (i.e. filter out) static tags nearby the conveyor belt, or tags moving on a random path different from that assumed for the conveyor belt (e.g. tags on a forklift moving close to the conveyor belt in a warehouse). Preliminary results of such a method extension have been presented in [41], where some parameters of the matching function are employed as input features of a classification algorithm.

\section{CONCLUSIONS}

The performance of a synthetic-array phase-based technique to locate items moving along a conveyor belt and equipped with UHF-RFID tags has been deeply analyzed and discussed, through both numerical and measured data. The localization technique can work with conventional wide-beam reader antennas, without requiring any hardware modification of the commercial reader. It has been demonstrated that typical geometrical and electrical parameters of UHF-RFID systems can allow for a localization accuracy of the order of a few centimeters, which is comparable with the size of many UHF tags. Design criteria relevant to the reader antenna-belt distance, the reader antenna beamwidth, and the observation spatial interval have also been discussed. Noteworthy, the observed robustness with respect to the multipath is actually intrinsic to the method, it being a global one. Indeed, the whole phase history is processed and matched, and not only a few samples as it is done in other methods, as those based on a phase-gradient or direction-of-arrival analysis. It is worth noting that the new synthetic-array phase-based technique can be easily applied to belts with an arbitrary path shape. Also, if enough readings are collected within the observation spatial interval, the tag can be localized in almost any point on the conveyor belt (not only when the tag passes right in front of the reader antenna). Finally, the good accuracy of the method is expected to be achievable also at longer ranges than the experimented one, conditioned to the operation of the reader, if a long enough observation spatial interval is available; this will be considered in future experimental research. 


\section{ACKNOWLEDGMENT}

The authors acknowledge the partial financial support by the "Fondazione Pisa", Pisa, Italy, under SARFID project (contract n. 204/11, 16/11/2012). Also, the authors would like to thank "DSU Toscana" for the permission to carry out the measurement campaign at one of the student restaurants of the University of Pisa.

\section{REFERENCES}

[1] N. Moayeri, J. Mapar, S. Tompkins, and K. Pahlavan, Special Issue on "Emerging opportunities for localization and tracking", IEEE Wireless Communication, vol. 18, no. 2, April 2011.

[2] M. Porretta, P. Nepa, G. Manara, and F. Giannetti, "Location, Location, Location: Use of Deterministic Propagation Models for Testing Wireless Networks Location Techniques", IEEE Vehicular Technology Magazine, vol. 3, no. 2, pp. 20-29, June 2008.

[3] K. Finkenzeller, RFID Handbook: Radio-Frequency Identification Fundamentals and Applications Hoboken, NJ: Wiley, 2004.

[4] R. Miesen, R. Ebelt, F. Kirsch, T. Schäfer, G. Li, H. Wang, and M. Vossiek, "Where is the Tag? History, Modern Concepts, and Applications of Locatable RFIDs", IEEE Microwave Magazine, vol. 12, no. 7, pp. S49-S63, December 2011.

[5] J. M. Chang, Y. P. Huang, and S. Liu, "Real-Time Location Systems and RFID", IEEE IT Professional, vol. 13, no. 2, pp. 12-13, March 2011.

[6] Z.-M. Liu, and R. R. Hillegass, "A 3 patch near field antenna for conveyor bottom read in RFID sortation application", Proc. of IEEE Antennas and Propagation Symposium, pp. 1043-1046, 2006.

[7] C.R. Medeiros, J.R. Costa, and C.A. Fernandes, "UHF RFID Smart Conveyor Belt with Confined Detection Range", IEEE Int. Symp. Ant. Prop., Charleston, SC, USA, June 5-9, 2009.

[8] A. Buffi, A. A. Serra, P. Nepa, H.-T. Chou, and G. Manara, "A Focused Planar Microstrip Array for 2.4 GHz RFID Readers", IEEE Trans. Antennas and Propagation, vol. 58, no. 5, pp 1536-1544, May 2010.

[9] R. Siragusa, P. Lemaitre-Auger, A. Pouzin, and S. Tedjini, "RFID tags localization along an axis using a tunable near-field focused circular-phase array antenna", 2011 XXXth URSI General Assembly and Scientific Symposium, August 2011.

[10] H.-T. Chou, T.-S. Hung, N.-N. Wang, H.-H. Chou, C. Tung, and P. Nepa, "Design of a Near-Field Focused Reflectarray Antenna for 2.4 GHz RFID Reader Applications", IEEE Trans. Antennas and Propagation, vol. 59, no. 3, pp. 1013-1018, March 2011.

[11] L. Catarinucci, R. Colella, L. Mainetti, L. Patrono, S. Pieretti, I. Sergi, and L. Tarricone, "Smart RFID Antenna System for Indoor Tracking and Behavior Analysis of Small Animals in Colony Cages", IEEE Sensors Journal, vol. 14, no. 4, pp. 1198-1206, April 2014.

[12] E. Di Giampaolo, and F. Martinelli, "A passive UHF-RFID system for the localization of an indoor autonomous vehicle", IEEE Trans. Industrial Electronics, vol. 59, no. 10, pp. 3961-3970, October 2012.

[13] Z. Zhang, Z. Lu, V. Saakian, X. Qin, Q. Chen, and L.R. Zheng, "Item-level indoor localization with passive UHF RFID based on tag interaction analysis", IEEE Trans. Industrial Electronics, vol. 61, no. 4, pp. 2122-2135, April 2014.

[14] Shao Shuai, and R.J. Burkholder, "Item-Level RFID Tag Location Sensing Utilizing Reader Antenna Spatial Diversity", IEEE Sensors Journal, vol. 13, no. 10, pp. 3767-3774, October 2013.

[15] L. Shangguan, Z. Li, Z. Yang, and M. Li, Y. Liu, “OTrack: Order tracking for luggage in mobile RFID systems", IEEE 2013 Proc. INFOCOM, pp. 30663074, April 2013.

[16] K. Chawla, and G. Robins, “An RFID-based object localization framework”, Proc. of Int. Journal on RFID Technology and Applications, Vol. 3, Nos. 1/2 2011, pp. 2-30.

[17] P. V. Nikitin, R. Martinez, S. Ramamurthy, H. Leland, G. Spiess, and K.V.S. Rao, "Phase Based Spatial Identification of UHF RFID Tags", 2010 IEEE Int. Conf. RFID, pp. 102-109, April 2010.

[18] V. Viikari, P. Pursula, and K. Jaakkola, "Ranging of UHF RFID Tag Using Stepped Frequency Read-Out", IEEE Sensors Journal, vol. 10, no. 9, pp. 1535-1539, September 2010.

[19] S. Sarkka, V.V. Viikari, M. Huusko, and K. Jaakkola, "Phase-Based UHF RFID Tracking With Nonlinear Kalman Filtering and Smoothing", IEEE Sensors Journal, vol. 12, no. 5, pp. 904-910, May 2012.

[20] E. Di Giampaolo, and F. Martinelli, "Mobile Robot Localization Using the Phase of Passive UHF RFID Signals", IEEE Trans. Industrial Electronics, vol. 61, no. 1, pp. 365-376, January 2014.

[21] Ma Yongtao, Zhou Liuji, Liu Kaihua, and Wang Jinlong, "Iterative Phase Reconstruction and Weighted Localization Algorithm for Indoor RFID-Based Localization in NLOS Environment”, IEEE Sensors Journal, vol. 14, no. 2, pp. 597-611, February 2014.

[22] M. Scherhaufl, M. Pichler, E. Schimback, D.J. Muller, A. Ziroff, and A. Stelzer, "Indoor Localization of Passive UHF RFID Tags Based on Phase-ofArrival Evaluation", IEEE Transactions on Microwave Theory and Techniques, vol. 61, no. 12, pp. 4724-4729, December 2013.

[23] Y. Zhang, M. G. Amin, and S. Kaushik, "Localization and Tracking of Passive RFID Tags Based on Direction Estimation," Int. Journal of Antennas and Propagation, vol. 2007, Article ID 17426.

[24] Lyngsoe Systems; P. C. F. Eggers, B. R. Yanakiev, and G. F. Pedersen, "System and method for wireless communications", US 20120182129 A1 patent.

[25] P. Nepa, F. Lombardini, and A. Buffi, "Method for determining the location of a moving RFID tag", EP 2533173 A1 patent.

[26] P. Nepa, F. Lombardini, and A. Buffi, "Location and tracking of UHF-RFID tags", Proc. of IEEE-APS APWC, pp. 1062-1065, Turin, Italy, September 12$16,2011$.

[27] P. Nepa, F. Lombardini, and A. Buffi, "Location and Tracking of Items Moving on a Conveyor Belt and Equipped with UHF-RFID Tags", IEEE Int. Symp. Antennas and Propagation, pp. 1-2, Chicago, USA, July 2012.

[28] A. Parr, R. Miesen, and M. Vossiek, "Inverse SAR approach for localization of moving RFID tags", 2013 IEEE Int. Conf. RFID, pp. 104-109, April-May 2013.

[29] G. Franceschetti, and R. Lanari, Eds., "Synthetic Aperture Radar Processing”, Boca Raton, FL: CRC Press, 1999.

[30] A. Buffi, A. Baroni, and P. Nepa, "Experimental Validation of Phase-Based Localization of UHF-RFID tags moving on a Conveyor Belt", Proc. of IEEE Int. Symp. Antennas and Propagation, pp. 2319-2320, July 7-13, 2013.

[31] F. Lombardini, F. Viviani, P. Nepa, and A. Buffi, "Robust Coherent Localization of Moving UHF-RFID Tags by a SAR-Like Approach," Proc. 10th European Conference on Synthetic Aperture Radar, EUSAR 2014, June 3-5, 2014, Berlin, Germany.

[32] R. Miesen, F. Kirsch, and M. Vossiek, "Holographic localization of passive UHF RFID transponders", 2011 IEEE Int. Conf. RFID, pp. 32-37, April 2011.

[33] G. Fornaro, F. Lombardini, and F. Serafino, "Three-dimensional Multipass SAR Focusing: Experiments with Long-term Spaceborne Data", IEEE Trans. Geoscience and Remote Sensing, vol. 43, no. 4, pp. 702-714, April 2005. 
[34] F. Lombardini, M. Pardini, G. Fornaro, F. Serafino, L. Verrazzani, and M. Costantini, "Linear and Adaptive Spaceborne Three-dimensional SAR Tomography: a Comparison on Real Data," IET Radar, Sonar \& Navigation, Special Issue, vol. 3, no. 4, pp. 424-436, August 2009.

[35] S. Haykin, and B. Van Veen, "Signal and Systems", New York, NY: John Wiley \& Sons, 1999.

[36] M. Pardini, F. Lombardini, and F. Gini, "The Hybrid Cramér-Rao Bound on Broadside DOA Estimation of Extended Sources in Presence of Array Errors," IEEE Trans. on Signal Processing, vol. 56, no. 4, pp. 1726-1730, April 2008.

[37] C. E. Shannon, "Communication in the presence of noise", Proc. Institute of Radio Engineers, vol. 37, no. 1, pp. 10-21, January 1949. Reprint as classic paper in: Proc. IEEE, vol. 86, no. 2, February 1998.

[38] F. Lombardini, "Optimum Absolute Phase Retrieval in Three-element SAR Interferometer", IEE Electronics Letters, vol. 34, no. 15, pp. 1522-1524, July 1998.

[39] D. Arnitz, U. Muehlmann, and K. Witrisal "Characterization and Modeling of UHF RFID Channels for Ranging and Localization", IEEE Trans. Antennas and Propagation, vol. 60, no. 5, pp. 2491-2501, May 2012.

[40] A. Buffi, P. Nepa, and G. Manara, "An indoor propagation model for development and testing of UHF-RFID tag localization techniques", Proc. of ICEAA, Turin, Italy, pp. 1012-1015, September 2013.

[41] A. Buffi, and P. Nepa, "A phase-based technique for discriminating tagged items moving through a UHF-RFID gate", IEEE RFID-TA Conference, Tampere, Finland, September 8-9, 2014. 